\title{
miR-205-3p promotes lung cancer progression by targeting APBB2
}

\author{
LING-BIN XU ${ }^{1}$, JIE XIONG $^{1}$, YA-HUI ZHANG ${ }^{2}$, YUN DAI $^{3}$, XIAO-PING REN ${ }^{1}$, \\ YA-JUAN REN ${ }^{1}$, DONG HAN ${ }^{1}$, SHENG-HONG WEI ${ }^{1}$ and MIN QI ${ }^{4}$ \\ ${ }^{1}$ Department of Pulmonary and Critical Care Medicine No. 2, Shaanxi Provincial People's Hospital, Xi'an, Shaanxi 710068;
${ }^{2}$ Department of Orthopaedics, Xi'an Daxing Hospital, Xi'an, Shaanxi 710016; ${ }^{3}$ Department of Thoracic Surgery and
${ }^{4}$ Imaging Center, Shaanxi Provincial People's Hospital, Xi'an, Shaanxi 710068, P.R. China
}

Received August 26, 2020; Accepted March 29, 2021

DOI: $10.3892 / \mathrm{mmr} .2021 .12227$

\begin{abstract}
Non-small cell lung cancer (NSCLC), a leading cause of cancer-associated mortality, has resulted in low survival rates and a high mortality worldwide. Accumulating evidence has suggested that microRNAs (miRs) play critical roles in the regulation of cancer progression and the present study aimed to explore the underlying mechanism of miR-205 in NSCLC. Reverse transcription-quantitative PCR was performed, which determined that miR-205 expression was upregulated in NSCLC, and the present study detected the upregulation of miR-205-3p in a number of NSCLC cell lines and NSCLC tissues. In addition, the mediation of amyloid $\beta$ precursor protein-binding family B member 2 (APBB2) by miR-205-3p was demonstrated. Moreover, miR-205-3p was predicted to directly target the 3 'untranslated region of $\mathrm{APBB} 2$, which was confirmed using a dual-luciferase reporter assay. It was found that lentivirus mediated-APBB2 knockdown could promote cellular viability and suppress apoptosis in NSCLC cells, as determined via MTT, TUNEL and flow cytometry assays. Thus, the current findings highlighted the potential promotive impact of miR-205-3p on NSCLC processes and may provide theoretical evidence for miR-205-3p as a potential clinical gene therapy target.
\end{abstract}

\section{Introduction}

Lung cancer is a major cause of mortality and has a survival rate of only $15.6 \%$ in all affected patients (1), although this rate can reach up to $55-80 \%$ in early-stage cases (2). In the United States, $>225,000$ new cases of lung cancer are diagnosed each year and $\sim 160,000$ deaths occur annually (3). Most patients with lung cancer $(\sim 85 \%)$ have non-small cell lung cancer (NSCLC) (4). Despite improvements in cancer diagnosis and

Correspondence to: Professor Min Qi, Imaging Center, Shaanxi Provincial People's Hospital, 256 Youyi Xilu Road, Xi'an, Shaanxi 710068, P.R. China

E-mail: minqivip@126.com

Key words: lung cancer, microRNA-205-3p, amyloid $\beta$ precursor protein-binding family B member 2, proliferation, apoptosis treatments, the 5-year survival rate has remained as low as $15 \%$ since the 1970s (5). Therefore, further investigations into the mechanisms of NSCLC development and progression are of the utmost importance for the diagnosis, prevention and treatment of this disease.

MicroRNAs (miRNAs/miRs), a class of endogenous non-coding RNAs, can mediate various gene expression levels by suppressing the translation or accelerating the decay of the target mRNA (6,7). Previous data have revealed that miRNAs can regulate $30 \%$ of mRNAs (8). Furthermore, miRNAs have important effects on various pathological processes, such as cancer cell proliferation, migration and differentiation, as well as patient survival $(9,10)$. Numerous dysregulated miRNAs, including miR-21, miR-100, miR-138 and miR-847, have been verified to be involved in NSCLC cell processes (11-14), which indicates that the aberrant expression of mRNA is associated with NSCLC. Moreover, miR-205 is significantly increased in NSCLC and has been demonstrated to serve as a biological marker for NSCLC (15). Su et al (16) observed that abnormal expression of miR-205 in endometrial cancer was closely associated with tumor proliferation and invasion. Another study reported that upregulated miR-205 expression could enhance the proliferation and the migration of cervical cancer cells (17). Collectively, these previous findings have suggested the potential promotive role of miR-205 on cancer processes. Despite of these findings, the role and underlying mechanisms of miR-205 in NSCLC remain largely unclarified.

Amyloid $\beta$ precursor protein-binding family B member 2 (APBB2) is an adaptor protein that belongs to the $\beta$-amyloid precursor protein-binding family $\mathrm{B}$. APBB 2 can bind to the cytoplasmatic domain of the $\beta$-amyloid precursor protein $(\beta \mathrm{APP})$. Thus, APBB2 may be involved in mediating APP processing (18). Lim et al (19) revealed that APBB2 overexpression promoted the formation of APP and reduced the response to apoptotic stimuli. Although the function of APBB2 is important, it remains unclear whether APBB2 could be regulated at the transcriptional level in NSCLC.

As stated in the aforementioned previous studies, both miR-205-3p and APBB2 may play crucial roles in the development of NSCLC, but the association between them and their regulatory roles in NSCLC remain unclear. In the current study, the expression of miR-205-3p in NSCLC was detected, followed by miR-205-3p biofunction analyses with MTT, TUNEL and flow cytometry assays. Following 
this, the potential downstream target of miR-205-3p, APBB2, was predicted and further investigated using a dual-luciferase reporter assay. In addition, the effect of the miR-205-3p/APBB2 axis on the proliferation and apoptosis of NSCLC was also explored. According to these investigation, the present study aimed to clarify the underlying mechanism of the miR-205-3p/APBB2 axis in NSCLC and provide some novel insights in the treatment of NCSCLC.

\section{Materials and methods}

Cell culture and tissue samples. The NSCLC cell lines A549, H1299, 95-D and H460, and the BEAS-2B normal human epithelial cell line were obtained from The Cell Bank of Type Culture Collection of The Chinese Academy of Sciences. The cells were cultured in Dulbecco's modified Eagle medium (DMEM; Gibco; Thermo Fisher Scientific, Inc.) supplemented with $10 \%$ fetal bovine serum (FBS; Gibco; Thermo Fisher Scientific, Inc.), $100 \mathrm{IU} / \mathrm{ml}$ penicillin (Shanghai Sangong Pharmaceutical Co., Ltd.) and $100 \mathrm{mg} / \mathrm{ml}$ streptomycin (Shanghai Sangong Pharmaceutical Co., Ltd.) at $37^{\circ} \mathrm{C}$ with $5 \% \mathrm{CO}_{2}$.

NSCLC tissue samples and adjacent normal tissues $(1 \mathrm{~cm}$ from tumor margin) were collected from 20 patients at the Shaanxi Provincial People's Hospital (Xi'an, China) between January and October 2019. All samples were snap-frozen in liquid nitrogen and maintained at $-80^{\circ} \mathrm{C}$. None of the patients had undergone chemotherapy or radiotherapy before tissue sampling. The NSCLC samples were obtained from 8 patients with advanced-stage disease (III and IV) and 12 patients with early-stage disease (I and II) who had provided informed consent before collecting; all pertinent diagnoses were conducted under the Revised International System for Staging Lung Cancer (20). This research was performed in accordance with the World Medical Association Declaration of Helsinki and was approved by the Institutional Review Board of the Shaanxi Provincial People's Hospital (approval no. SXRMYY-2019-015). Written informed consent was obtained from all patients.

Cell transfection. miR-205-3p inhibitor (50 nM; 5'-GAUUUC AGUGGAGUGAAGUUC-3') and negative controls (miR-NC; $50 \mathrm{nM}$; 5'-UCACAACCUCCUAGAAAGAGUAGA-3') were obtained from Changzhou Ruibo Bio-Technology Co., Ltd. Two specific small interfering (si)RNAs against APBB2 (50 nM; APBB2-siRNA1, 5'-AUUUUCCAUCCAGCAAAU GUU-3'; APBB2-siRNA2, 5'-UUGCUUAAAUUAUUG UCAGCC-3') and a scrambled NC (50 nM; si-NC, 5'-CAC UGAUUUCAAAUGGUGCUAUU-3') were synthesized by Shanghai GenePharma Co., Ltd. All transfections were performed using Lipofectamine ${ }^{\circledR} 2000$ (Invitrogen; Thermo Fisher Scientific, Inc.) in accordance with the manufacturer's protocol. Briefly, $3 \times 10^{5}$ cells/well were seeded in a 6 -well plate and were incubated until they reached $\geq 70 \%$ confluence. Cells were then transfected with the miR-205 inhibitor, APBB2 siRNA1, APBB2 siRNA2 and their respective NCs using Lipofectamine 2000 reagent. After $72 \mathrm{~h}$, the cells were harvested for further investigation. The efficiency was validated by reverse transcription-quantitative PCR (RT-qPCR) or western blotting.
RT-qPCR. Total RNA from tissues and cell lines was isolated using TRIzol ${ }^{\circledR}$ reagent (Invitrogen; Thermo Fisher Scientific, Inc.). cDNA was synthesized using the PrimeScript RT reagent kit (Takara Bio, Inc.) according to the manufacturer's protocol. qPCR was performed using the SYBR ${ }^{\circledR}$ Green PrimeScript ${ }^{\mathrm{TM}}$ PLUS RT-PCR kit (Takara Bio, Inc.). The reaction conditions were as follows: Initial denaturation at $95^{\circ} \mathrm{C}$ for $30 \mathrm{sec}$; followed by 40 cycles of denaturation at $95^{\circ} \mathrm{C}$ for $15 \mathrm{sec}$, annealing at $60^{\circ} \mathrm{C}$ for $25 \mathrm{sec}$ and extension at $72^{\circ} \mathrm{C}$ for $1 \mathrm{~min}$; and a final extension step at $72^{\circ} \mathrm{C}$ for $10 \mathrm{~min} . \beta$-actin (mRNA) and U6 (miRNA) were used as the internal controls and the relative expression of genes was calculated using $2^{-\Delta \Delta C q}$ method (21). The following primers were used: miR-205-3p forward, 5'-CTTGTCCTTCATTCCACCGGA-3' and reverse, 5'-TGC CGCCTGAACTTCACTCC-3'; APBB2 forward, 5'-CACAGA GAAGAGTCTGGCCC-3' and reverse, 5'-AGGTTGCTTGTG ACAGGTCC-3'; $\beta$-actin forward, 5'-GATGGACTCTGGTGA TGGTGTGAC-3' and reverse, 5'-TTTCTCTTTCGGCTG TGGTGGTG-3'; and U6 forward, 5'-CTCGCTTCGGCAGCA CA-3' and reverse, 5'-AACGCTTCACGAATTTGCGT-3'.

MTT assay. An MTT assay was used to analyze cell viability. Transfected cells were seeded into 96-well plates at a density of $1.0 \times 10^{4}$ per well and cultured at $37^{\circ} \mathrm{C}$ for 24,48 and $72 \mathrm{~h}$. After being mixed with $0.5 \mathrm{mg} / \mathrm{ml}$ MTT solution (Sigma; Merch $\mathrm{KGaA}$ ) for $4 \mathrm{~h}$ and co-culturing with $100 \mu \mathrm{l}$ DMSO for $10 \mathrm{~min}$ at $37^{\circ} \mathrm{C}$, the optical density values were determined at $490 \mathrm{~nm}$.

TUNEL staining. A DeadEnd Fluorometric TUNEL Detection system (Promega Corporation) was used to evaluate apoptosis, according to the manufacturer's protocol. Briefly, $3 \times 10^{5}$ cells were seeded onto a cover slip in a six-well plate and attached overnight. Then, cells were treated as indicated followed by $4 \%$ formaldehyde-PBS fixation for $15 \mathrm{~min}$ at room temperature, after which they were permeabilized with $0.2 \%$ Triton X-100 in PBS for another 10 min under the same conditions. After being washed twice with PBS, the cells were incubated with a fluorometric terminal deoxytransferase mixture at $37^{\circ} \mathrm{C}$ for $1 \mathrm{~h}$ and then washed twice with PBS buffer. Then, cells on slips were mounted using DAPI-containing mounting medium (Vector Laboratories, Inc.) according to the manufacturer's protocol. Fluorescence images were captured in at least five views using a Nikon Eclipse Ti-E fluorescence microscope (Nikon Corporation).

EdU assay. The effect of miR-205-5p on cell proliferation was detected using EdU staining (Guangzhou RiboBio Co., Ltd.). Briefly, $3 \times 10^{5}$ cells were seeded onto a cover slip in a six-well plate and maintained overnight followed by the indicated treatments. Then, cells were incubated with $30 \mu \mathrm{M}$ EdU contained in serum-free DMEM. After washing in PBS three times, cells were fixed with $4 \%$ polyformaldehyde in PBS at room temperature for $30 \mathrm{~min}$ in the dark. Following this, cells were incubated with Apollo staining solution and Hoechst 33342 at room temperature for $30 \mathrm{~min}$. Finally, staining images were obtained using a Nikon Eclipse Ti-E fluorescence microscope (Nikon Corporation). Five random field of views were chosen to count the number of positively stained cells, and proliferation was assessed using the percentage of EdU-positive cells relative to the total cell number. 
A


B



D

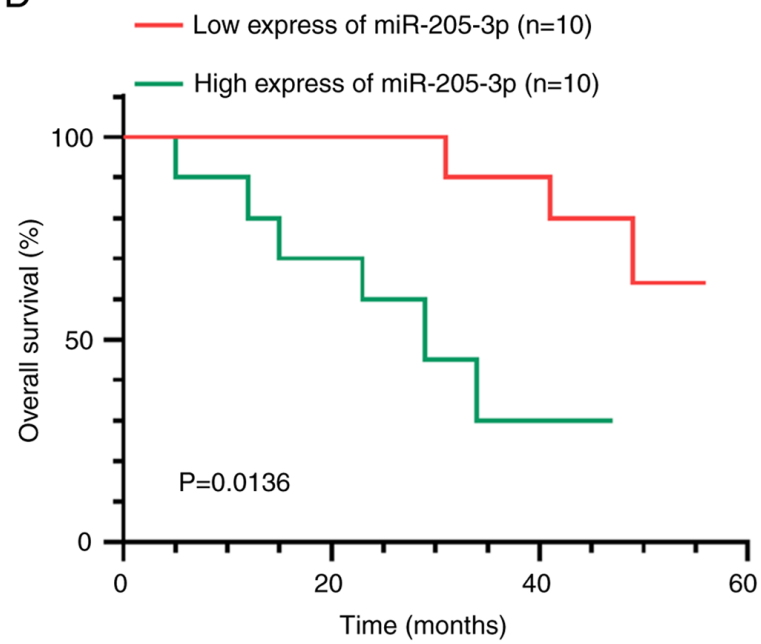

Figure 1. miR-205-3p expression is upregulated in NSCLC tissues and cell lines and is associated with disease progression in patients with NSCLC. (A) miR-205-3p expression in 20 NSCLC tissues and paired adjacent normal lung tissues was detected by reverse transcription-quantitative PCR. (B) miR-205-3p expression in NSCLC cell lines and normal human lung epithelial cells (BEAS-2B). (C) Expression level of miR-205-3p was compared between eight patients with advanced-stage disease (III and IV) and 12 patients with early-stage disease (I and II). (D) A total of 20 patients with NSCLC were classified into the high expression group $(n=10)$ and low expression group $(n=10)$, based on the median miR-205-3p expression in tumor tissues. The Kaplan-Meier overall survival curve of the two patient groups is presented; a log-rank test was used for survival analysis. Data are presented as the mean \pm SD of three independent experiments. ${ }^{*} \mathrm{P}<0.05,{ }^{* *} \mathrm{P}<0.01$. miR, microRNA; NSCLC, non-small cell lung cancer.

Flow cytometric analysis of apoptosis. Flow cytometry was performed to determine the alteration of apoptosis. After harvesting, the cells were washed with PBS solution twice, followed by staining with a Annexin V-FITC/PI Kit in the dark (Sigma-Aldrich; Merck KGaA), according to the manufacturer's instructions. Apoptosis was analyzed using a BD FACSCalibur flow cytometer (BD Biosciences) with Cell Quest software (version 5.0; BD Biosciences). The apoptotic rate was counted as the percentage of early apoptosis plus the percentage of late apoptosis.

Western blotting. After reaching 80-90\% confluence, cells were lysed in RIPA buffer (BioVision, Inc.) solution with protease inhibitor and phosphatase inhibitor cocktail (Sigma-Aldrich; Merck KGaA), followed by centrifugation at $10,000 \mathrm{x} \mathrm{g}$ at $4^{\circ} \mathrm{C}$ for $10 \mathrm{~min}$. Then, the supernatant of cell lysate was collected and quantified using BCA method (Thermo Fisher Scientific, Inc.). Total protein $(25 \mu \mathrm{g})$ was separated via $10 \%$ SDS-PAGE, and subsequently transferred to a nitrocellulose membrane (EMD Millipore). Then, the membrane was blocked in $10 \%$ dried milk and $0.1 \%$ BSA (Fraction V; Sigma-Aldrich; Merck KGaA) in PBS for $1 \mathrm{~h}$ at room temperature, followed by incubation at $4^{\circ} \mathrm{C}$ overnight with primary antibodies against $\operatorname{APBB} 2(1: 2,500$; cat. no. PA5-54816; Thermo Fisher Scientific, Inc.), Bax (1:2,500; cat. no. MA5-13994; Sigma-Aldrich; Merck KGaA), Bcl-2 (1:1,000; cat. no. 13-8800; Thermo Fisher Scientific, Inc.) and $\beta$-actin (1:5,000; cat. no. MA1-744; Thermo Fisher Scientific, Inc.). Then, membranes were incubated with HRP-conjugated secondary goat anti-rabbit (1:20,000; cat. no. G-21234; Sigma-Aldrich; Merck KGaA) or goat anti-mouse antibody (1:20,000; cat. no. G-21040; Sigma-Aldrich; Merck KGaA) at room temperature for $1 \mathrm{~h}$. Protein bands on membranes were visualized using the enhanced chemiluminescence (ECL) method (Pierce; Thermo Fisher Scientific, Inc.). $\beta$-actin was used as the internal control and protein expression levels were semi-quantified using Quantity One 4.6 software (Bio-Rad Laboratories, Inc.). 
Prediction of miR-205-3p target genes. The downstream target genes of miR-205-3p were predicted using miRDB (http://mirdb.org/index.html) and TargetScan (version 7.0; http://www.targetscan.org/vert_72/). Cell cycle arrest-associated genes were obtained from Gene Ontology (GO; http://geneontology.org). Then, the overlapping genes among miRDB target genes, TargetScan target genes and GO cell cycle arrest-associated genes were isolated using Venn analysis (http://bioinformatics.psb.ugent.be/webtools/Venn/).

Luciferase assay. The amplified wild-type (WT) APBB2-3'untranslated region (UTR) and mutant (MUT) APBB2-3'UTR, which was produced using a Q5 Site-Directed Mutagenesis kit (cat. no. E0554S; New England BioLabs, Inc.), according to the manufacturer's protocol, were cloned into pGL3 luciferase vectors (Promega Corporation). The cells were co-transfected with $10 \mathrm{nM} \mathrm{miR-205-3p} \mathrm{inhibitor} \mathrm{(or} \mathrm{miR-NC)}$ and luciferase reporter vectors (200 ng/well) of the WT or MUT type 3'UTR of the APBB2 gene using Lipofectamine 2000 (Invitrogen; Thermo Fisher Scientific, Inc.) according to the manufacturer's instructions. Subsequently, the Dual-Luciferase Reporter Assay kit (Promega Corporation) was used to detect the relative luciferase activities $48 \mathrm{~h}$ after the transfections, according to the manufacturer's protocols. Renilla luciferase activity was used for normalization against firefly luciferase activity.

In vivo tumor biology. The present study experiments were performed at the Shaanxi Provincial People's Hospital; all experiments were performed following the Guidelines of Animal Use and Care Committee of the Shaanxi Provincial People's Hospital, and were approved by the Institutional Animal Care and Use Committee of Shaanxi Provincial People's Hospital. C57BL/6 mice (age, 6-8 weeks old; weight, $20 \pm 2 \mathrm{~g} ; \mathrm{n}=10$ ) were purchased from Shanghai SLAC Laboratory Animal Co., Ltd., maintained in the animal facility of the Shaanxi Provincial People's Hospital at room temperature $\left(22 \pm 1^{\circ} \mathrm{C}\right)$ with humidity of $55 \pm 2 \%$ and a $12 / 12$-h light/dark cycle, with access to food and water ad libitum. Briefly, $2 \times 10^{6}$ A549 cells in $0.2 \mathrm{ml}$ PBS were inoculated subcutaneously in the right flank of each mouse. After 7 days, the mice were intravenously injected with $100 \mu$ l antagomir NC or miR-205-3p antagomir (5 nmol) in the tail. Tumor volume was assessed using a slide caliper every 5 days and calculated using the following formula: Volume=length $\mathrm{x} \operatorname{width}^{2} / 2$. At 25 days after inoculation, all mice were anaesthetized with an intramuscular injection of $50 \mathrm{mg} / \mathrm{kg}$ ketamine mixed with $5 \mathrm{mg} / \mathrm{kg}$ xylazine (22). Then, mice were euthanized by cervical dislocation, and the tumor volume was measured. miR-205-3p expression levels were determined by RT-qPCR and western blot analysis was used to detect the APBB2 protein expression levels in tumors. Ki67 was detected by immunofluorescence.

Immunofluorescence. Fresh tumor samples were washed with PBS to remove blood, and dehydrated with gradient sucrose solution. Then, samples were embedded in opti-MEM cutting temperature compound and frozen at $-80^{\circ} \mathrm{C}$ to form hardened blocks. Following this, tumor samples were cut into $6-\mu \mathrm{m}$ thick slides, fixed in $100 \%$ acetone at $4^{\circ} \mathrm{C}$ for $5 \sim 10 \mathrm{~min}$, permeabilized in $0.025 \%$ Triton X-100 with PBS, blocked by
Table I. Clinicopathological characteristics of patients with non-small cell lung cancer at the time of first diagnosis.

\begin{tabular}{lc}
\hline Clinicopathological characteristics & Patients $(\mathrm{n}=20)$ \\
\hline Age, years & $58.73 \pm 10.22$ \\
BMI, kg/m² & $22.14 \pm 2.43$ \\
Sex & \\
Male & $11(55)$ \\
Female & $9(45)$ \\
TNM stage & \\
I-II & $12(60)$ \\
III-IV & $8(40)$ \\
Tumor size, cm & $13(65)$ \\
$>5$ & $7(35)$ \\
$\leq 5$ & \\
Smoking history & $14(70)$ \\
Yes & $6(30)$ \\
No & \\
\hline
\end{tabular}

Data are presented as the mean $\pm \mathrm{SD}$ or $\mathrm{n}(\%)$.

PBS with $10 \%$ goat serum (cat. no. C0265; Beyotime Institute of Biotechnology) and 1\% BSA at room temperature for $1 \mathrm{~h}$. Following this, slides were incubated with rabbit anti-Ki67 antibody (1:500; cat. no. ab92742; Abcam) at $4^{\circ} \mathrm{C}$ in a humid room overnight. After rinsing with PBS three times, slides were incubated with goat anti-rabbit secondary antibody (Alexa Fluor $^{\circledR} 488$; 1:1,000; cat. no. ab150077; Abcam) at room temperature for $1 \mathrm{~h}$. Then, slides were incubated with PI $(100 \mu \mathrm{g} / \mathrm{ml}$; Sigma-Aldrich; Merck KGaA) at room temperature for $15 \mathrm{~min}$. Subsequently, slides were mounted with glycerin (Sangon Biotech Co., Ltd.) and stained images were collected using a Nikon Eclipse Ti-E fluorescence microscope (Nikon Corporation).

Statistical analysis. All data were collected in triplicate and presented as the mean $\pm \mathrm{SD}$. A paired Student's t-test was used for statistical analysis of RT-qPCR results of NSCLC tissues and their adjacent tissues. An unpaired Student's t-test or one-way ANOVA followed by Tukey's post hoc test was applied to the rest of the data to evaluate statistical differences. Kaplan-Meier analysis followed by a log-rank test was used to compare the overall survival between the two patient groups. Statistical analysis was performed using GraphPad Prism 6.0 (GraphPad Software, Inc.). P $<0.05$ was considered to indicate a statistically significant difference.

\section{Results}

miR-205-3p is upregulated in NSCLC tissues and cell lines. RT-qPCR was used to evaluate the expression levels of miR-205-3p in normal adjacent lung tissues and NSCLC tissues from patient samples. miR-205-3p expression was significantly higher in the 20 NSCLC tissues compared with the normal adjacent tissues (Fig. 1A). NSCLC cell lines, 

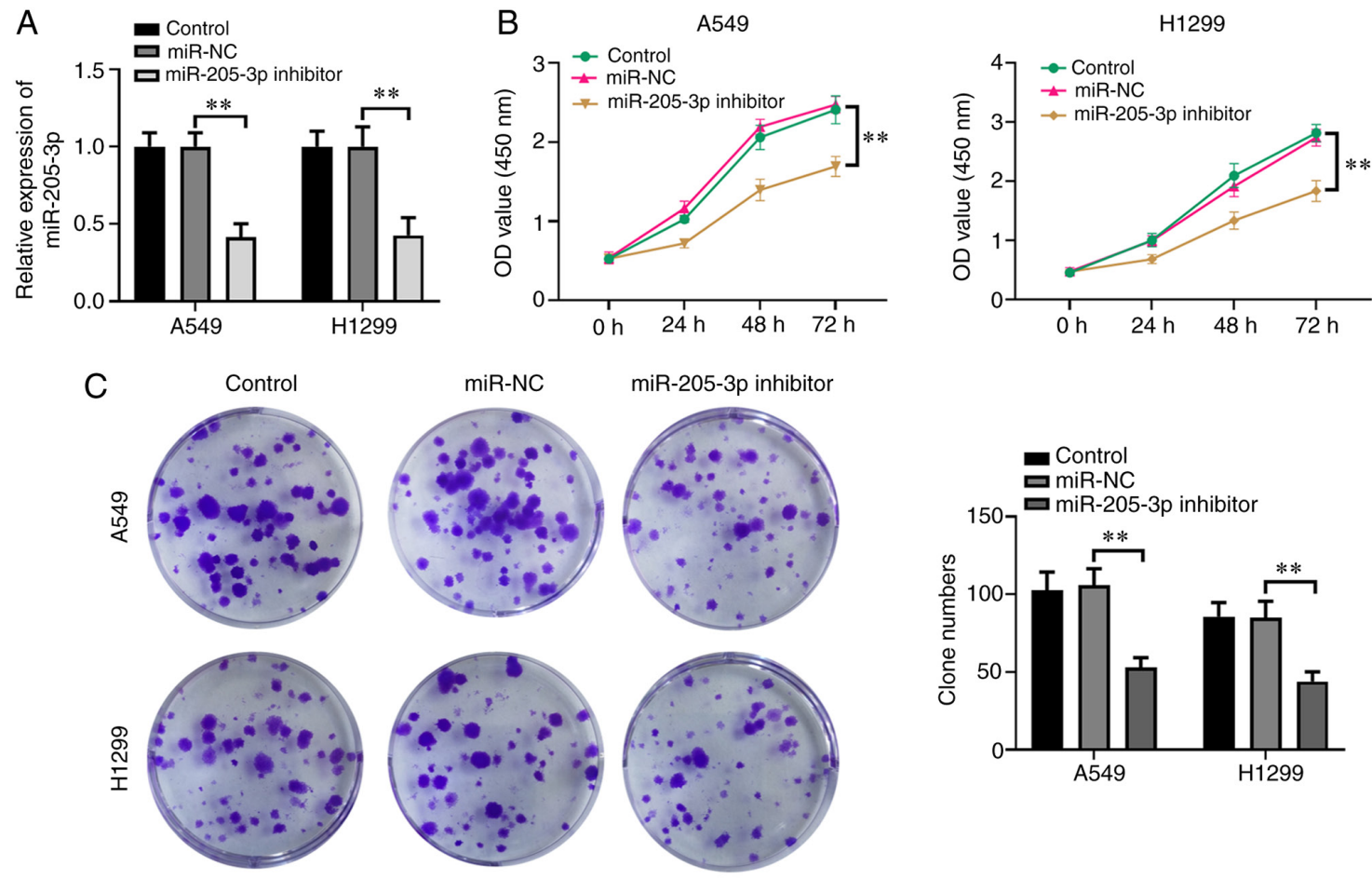

Figure 2. Effects of miR-205-3p knockdown on the proliferation of H1299 and A549 cells. (A) Reverse transcription-quantitative PCR analysis of miR-205-3p expression in A549 and H1299 cells transfected with the miR-205-3p inhibitor or miR-NC. (B) Effects of miR-205-3p knockdown on cell viability was evaluated using an MTT assay. (C) A colony formation assay was performed to assess the effects of miR-205-3p knockdown on cell proliferation. Data are presented as the mean $\pm \mathrm{SD}$ of three independent experiments. ${ }^{* *} \mathrm{P}<0.01$. miR, microRNA; NC, negative control; OD, optical density.

including A549, H1299, 95-D, H460 and H292 cells, and the BEAS-2B normal lung epithelial cells were used for further investigation. As presented in Fig. 1B, miR-205-3p expression was significantly elevated in NSCLC cell lines compared with that in the normal lung epithelial cells.

To investigate the variations in miR-205-3p expression, eight patients with advanced-stage disease (III and IV) and 12 patients with early-stage disease (I and II) were examined. It was revealed that miR-205-3p expression was significantly elevated in advanced stages compared with early stages (Fig. 1C). The 20 patients with NSCLC were divided into two groups based on their miR-205-3p expression levels in relation to the median value (cut-off, 1.26) as follows: Low expression $(n=10)$ and high expression $(n=10)$ miR-205-3p groups. These groups were followed-up for 50 months to obtain survival information. The results demonstrated that the patients in the low miR-205-3p expression group displayed higher survival rates compared with those in the high expression group (Fig. 1D). The characteristics of the patients with NSCLC are listed in Table I.

miR-205-3p knockdown reduces the proliferation of NSCLC cells. A miR-205-3p inhibitor was used to investigate the effects of miR-205-3p on H1299 and A549 NSCLC cell proliferation. A549 and H1299 cells were chosen for this assay as they showed the highest expression of miR-205-3p among the NSCLC cell lines. Compared with the control and miR-NC treatment groups, miR-205-3p expression was significantly lower in the miR-205-3p inhibitor-transfected group (Fig. 2A). The MTT assay was performed to examine the effects of miR-205 on H1299 and A549 cell proliferation. As presented in Fig. 2B, the miR-205-3p inhibitor-mediated knockdown of miR-205-3p significantly decreased cell viability at $72 \mathrm{~h}$ post-transfection. A colony formation assay was also conducted to analyze the possible effects of miR-205-3p knockdown on cell proliferation. The results indicated that the miR-205-3p inhibitor led to fewer clones compared with the control groups (Fig. 2C).

To further validate the function of miR-205-3p, BEAS-2B normal human lung epithelial cells were transfected with miR-205-3p mimics or the inhibitor (Fig. S1 A and B). It was found that neither the miR-205-3p mimics or inhibitors altered cell proliferation in BEAS-2B cells (Fig. S1C).

miR-205-3p knockdown accelerates the apoptosis of NSCLC cells. TUNEL staining was used to determine the potential impact of miR-205-3p knockdown on apoptosis in A549 and H1299 cells. Compared with the control and miR-NC groups, A540 and H1299 cells transfected with the miR-205-3p inhibitor had a high number of cells undergoing apoptosis, as identified by TUNEL staining (Fig. 3A). After being transfected for $48 \mathrm{~h}$, the apoptotic rates in the A549 and H1299 cells were also analyzed with PI and Annexin V/FITC staining (Fig. 3B and C). This assessment revealed that miR-205-3p knockdown significantly increased the apoptotic rates in the miR-205-3p inhibitor-transfected group compared with the controls.

Subsequently, the protein expression levels of cleaved caspase-3, which is closely associated with apoptosis, were investigated by western blotting. As shown in Fig. 3D, the results suggested that miR-305-3p knockdown could significantly increase cleaved caspase-3 expression in both NSCLC 
A


B



A549


$\mathrm{H} 1299$




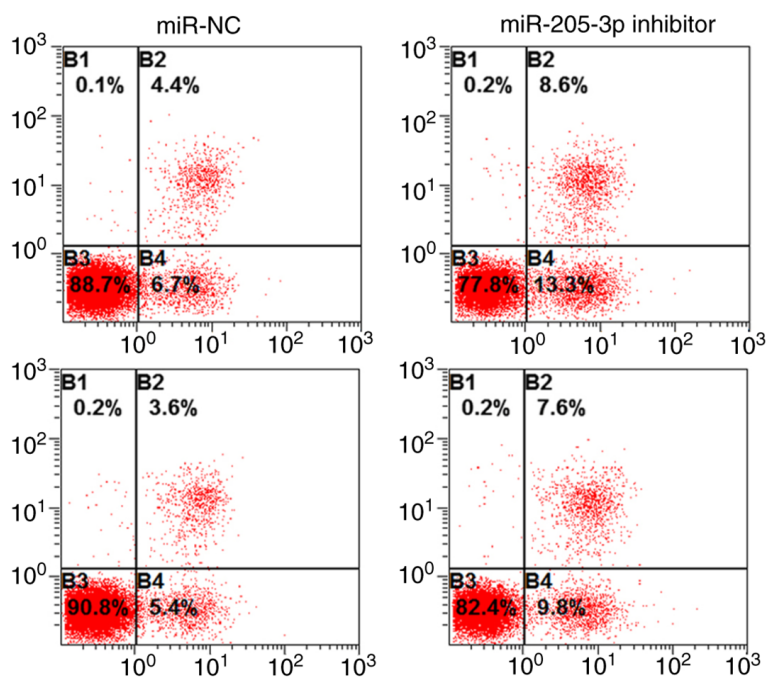

Annexin V/FITC

C


Figure 3. Effects of miR-205-3p knockdown on the apoptosis of H1299 and A549 cells. (A) TUNEL staining in A549 and H1299 cells transfected with the miR-205-3p inhibitor or the inhibitor miR-NC. Scale bar, $100 \mu \mathrm{m}$. (B) Representative flow cytometry dot plots and (C) graphs analyzing the apoptotic rates of A549 and H1299 cells transiently transfected with the miR-205-3p inhibitor or miR-NC. (D) Western blot analysis of the apoptosis-related protein cleaved caspase-3 in H1299 and A549 cells. Data are presented as the mean $\pm \mathrm{SD}$ of three independent experiments. ${ }^{* *} \mathrm{P}<0.01$. miR, microRNA; NC, negative control.

cell lines, which confirmed the outcomes of the TUNEL staining and Annexin V/PI staining.

$A P B B 2$ is a target gene of miR-205-3p. miRNAs can inhibit the biological functions of target genes by regulating gene expression (23). Moreover, each miRNA can target thousands of genes. The potential targets of miR-205-3p were predicted using miRDB and TargetScan. Then, cell cycle-associated genes targeted by miR-205-3p were isolated using Venn analysis based on miRDB target genes, target genes predicted using TargetScan, and cell cycle-associated genes found using GO (GO:0007050, 'cell cycle arrest'). As shown in Fig. S2, a single target gene in the center was identified among the three programs, APBB2. Thus, it was hypothesized that miR-205-3p may inhibit APBB2 



Figure 4. APBB2 is a target gene of miR-205-3p. (A) Predicted miR-205-3p target site in the 3'UTR of APBB2. (B) A549 and H1299 cells were co-transfected with miR-NC or miR-205-3p inhibitor and luciferase reporter vectors containing the WT or the MUT 3'UTR of APBB2. Relative luciferase activity was analyzed 48 h later. ${ }^{*}<<0.05$ vs. miR-NC. (C) Reverse transcription-quantitative PCR results of APBB2 mRNA expression in NSCLC cell lines (95-D, A549, H1299, H292 and H460) and normal human lung epithelial cells (BEAS-2B). A549 and H1299 cells were transfected with the miR-205-3p inhibitor, miR-NC or left untreated, and APBB2 (D) mRNA and (E) protein expression levels were analyzed. Data are presented as the mean \pm SD of three independent experiments. ${ }^{* *} \mathrm{P}<0.01$. miR, microRNA; NC, negative control; WT, wild-type; MUT, mutant; APBB2, amyloid $\beta$ precursor protein-binding family B member 2; UTR, untranslated region.

expression by binding to the 3'UTR. Moreover, APBB2 was found to have potential binding ability with miR-205-3p at the 3'UTR site (Fig. 4A). Therefore, the WT or MUT 3'UTR of APBB2 was inserted downstream of the firefly luciferase gene to confirm this hypothesis using a dual-luciferase assay (Fig. 4B). miR-205-3p-transfected A549 and H1299 cells were co-transfected with WT 3'UTR or MUT 3'UTR of APBB2 plasmid, and relative luciferase expression data were obtained after $48 \mathrm{~h}$. It was found that the miR-205-3p inhibitor could significantly increase WT 3'UTR luciferase activity compared with the miR-NC group, and no significant differences were identified between miR-NC and MUT 3'UTR (Fig. 4B).
Based on the aforementioned findings, RT-qPCR was conducted to further examine the potential alteration of APBB2 expression between normal human lung epithelial cells (BEAS-2B) and NSCLC cell lines (95-D, A549, H1299, H292 and H460). The data revealed that APBB2 mRNA expression levels in the NSCLC cell lines were significantly lower compared with that in BEAS-2B cells (Fig. 4C). As shown in Fig. 4D and E, after being transfected with the miR-205-3p inhibitor, the mRNA and protein expression levels of APBB2 were significantly increased in the A549 and H1299 cells. These results indicated that APBB2 may be a direct target of miR-205-3p in NSCLC. 



E
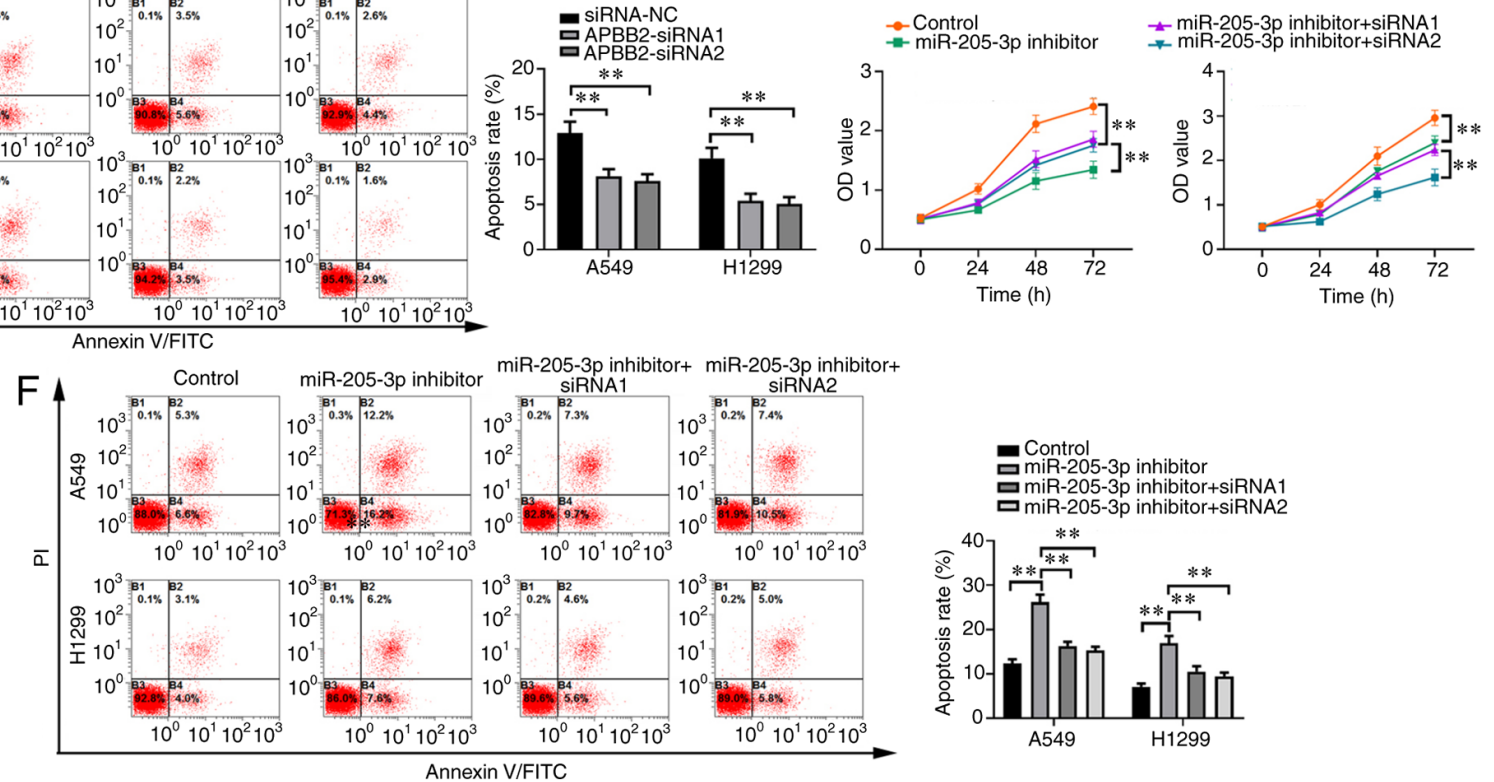

Figure 5. Effects of APBB2 on $\mathrm{H} 1299$ and A549 cell proliferation and apoptosis are regulated by miR-205-3p. APBB2 (A) mRNA and (B) protein expression in A549 and H1299 cells after transfection with APBB2-siRNA1, APBB2-siRNA2 or siRNA-NC. (C) Proliferation and (D) apoptosis analysis were performed in A549 and H1299 cells transfected with APBB2-siRNAs or si-NC. (E) Proliferation and (F) apoptosis of A549 and H1299 cells co-transfected with either APBB2-siRNA and the miR-205-3p inhibitor. Data are presented as the mean $\pm \mathrm{SD}$ of three independent experiments. ${ }^{* *} \mathrm{P}<0.01$. miR, microRNA; NC, negative control; APBB2, amyloid $\beta$ precursor protein-binding family B member 2; siRNA, small interfering RNA; OD, optical density.

Knockdown of APBB2 promotes cell proliferation. APBB2 genes were knocked down through transfection with APBB2-siRNA1 or APBB2-siRNA2, which were found to significantly decrease APBB2 mRNA and protein expression levels (Fig. 5A and B, respectively). After being transfected with either APBB2-siRNA, proliferation of the A549 and H1299 cells was significantly enhanced, whereas the apoptotic rates were decreased, compared with that of the siRNA-NC group (Fig. 5C and D). Furthermore, A549 and H1299 cells were co-transfected with either APBB2-siRNA and the miR-205-3P inhibitor. It was found that APBB2-siRNA partially alleviated miR-205-3p inhibitor-induced suppression of cell proliferation and, to a certain degree, reversed the promotion of apoptosis in both cell lines (Fig. 5E and F).

miR-205-3p antagomir inhibits lung cancer growth and promotes apoptosis in mouse xenografts. miR-205-3p-medidated tumor suppression at the tissue level was investigated using a miR-205-3p antagomir administered to mouse xenograft models subcutaneously injected with A549 cells. As shown in Fig. 6A and B, tumors harvested from mice treated with miR-205-3p antagomir were smaller in size and weight compared with those of the antagomir NC group. RT-qPCR results demonstrated that the miR-205-3p antagomir could significantly decrease miR-205-3p expression (Fig. 6C), as well as promote APBB2 expression in tumors. Moreover, the miR-205-3p antagomir induced a significant decrease in Bcl-2 and a significant increase in Bax protein expression levels (Fig. 6D). It was also identified that APBB2 protein expression was upregulated after injection with miR-205-3p antagomir in the same tumor issues (Fig. 6D). Furthermore, the miR-205-3p antagomir could markedly inhibit cell proliferation in the tumor, with Ki67 as the index (Fig. 6E).

\section{Discussion}

Previous studies have investigated the potential effects of the aberrant expression levels of miRNAs on tumor development (24-26). Given the close association between miRNAs and tumors, accumulating evidence has revealed that modulating miRNA expression could be an effective novel therapeutic management of tumors (27). The present results indicated that miR-205-3p knockdown could significantly inhibit tumor growth and the viability of NSCLC cells, as well as promote the apoptotic rate, at least partially, by targeting APBB2, a tumor suppressor. 
A
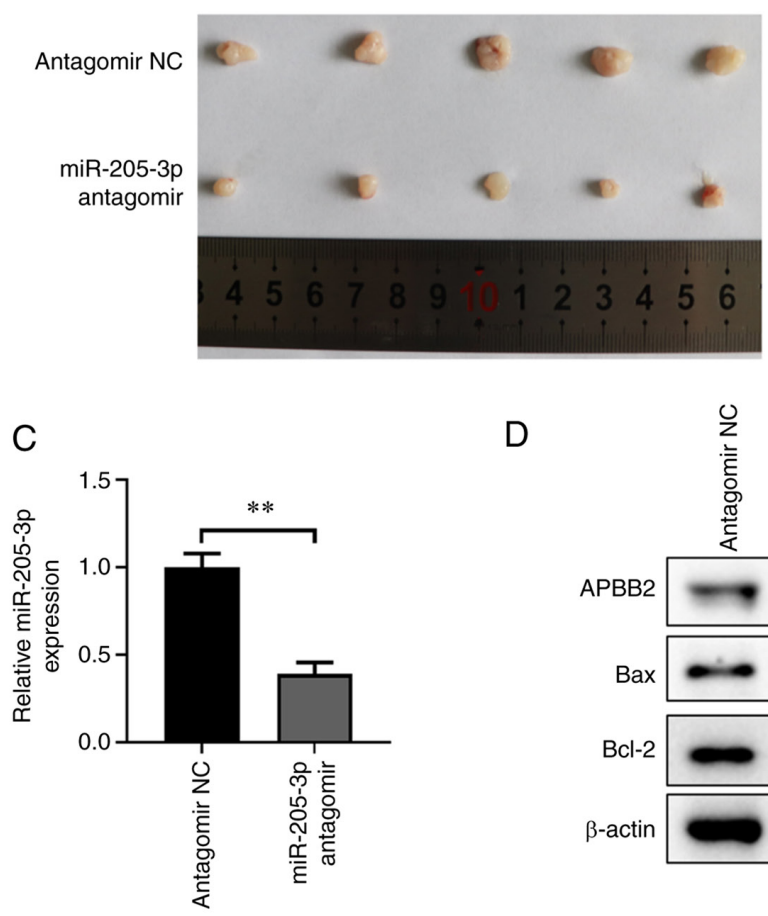

$\mathrm{E}$


D



$\mathrm{Pl}$


B


Figure 6. miR-205-3p antagomir inhibits lung cancer growth and promotes apoptosis in mouse xenografts. (A) Representative images of excised xenograft tumors and (B) tumor volumes from mice treated with the antagomir NC or miR-205-3p antagomir. (C) Reverse transcription-quantitative PCR analysis was used to detect miR-205-3p expression in tumors. (D) Western blot analysis was used to detect APBB2, Bax and Bcl-2 protein expression levels in tumors. ${ }^{*} \mathrm{P}<0.05,{ }^{* *} \mathrm{P}<0.01$ vs. antagomir NC. (E) Representative images of Ki67 staining; nuclei were stained with DAPI. Scale bar, $100 \mu$ m. Data are presented as the mean \pm SD of three independent experiments. miR, microRNA; NC, negative control; APBB2, amyloid $\beta$ precursor protein-binding family B member 2 .

miR-205, which is located on the second intron of the LOC642587 locus in chromosome 1, serves an important role in various types of cancer (28-30). In brief, as a tumor suppressor, miR-205 overexpression can notably inhibit tumor development (31). Moreover, the expression level of miR-205 is closely associated with the presence of fewer melanoma tissues, and a lower expression results in worse clinical outcomes $(32,33)$. Conversely, miR-205 can promote cancer formation in some cancer types. For example, Niu et al (34) reported that miR-205 was upregulated in ovarian cancer, and its expression level was positively correlated with advanced clinical stages. The different roles of miR-205 may be associated with the targets in different cancer microenvironments. Thus, the aim of the present research was to determine the potential relative expression levels of miR-205-3p in cancer and normal adjacent tissues from patients with NSCLC. The present study initially revealed that miR-205-3p expression in lung tumor tissues was significantly higher compared with that in adjacent normal tissues. Moreover, miR-205-3p overexpression was associated with advanced stages (III and V) and a shorter overall survival for patients in the clinic. Conversely, the knockdown of miR-205-3p using a miR-205-3p inhibitor lead to increased apoptosis in H1299 and A549 cells and a smaller tumor volume in mice. Collectively, the current data suggested that miR-205-3p served 
an essential role in NSCLC tumor development and could accelerate lung cancer progression.

The present study further investigated the underlying mechanism of the promotion function of miR-205-3p on tumor development. APBB2 is an adaptor protein that binds to the cytoplasmatic domain of $\beta$ APP (35), and it was predicted to be the target of miR-205-3p through binding at the 3'UTR. To verify this prediction, the present study detected the altered expression level of APBB2 by transfecting the miR-205-3p inhibitor into NSCLC cells and normal human lung epithelial cells. The current data revealed that there were significant differences in APBB2 expression between the NSCLC cells and normal cells. Moreover, APBB2 mRNA and protein expression was significantly elevated by the miR-205-3p inhibitor in both cellular and tissue models compared with the control-treated group. Collectively, these data suggested that APBB2 was a target gene of miR-205-3p at the 3'UTR sites, and this finding was supported by the luciferase reporter assay results.

However, there are some limitations of the present study. First, although it was documented that miR-205-3p could regulate the process of lung cancer via targeting APBB2, the detailed mechanisms of how APBB2 modulates cancer cell behaviors need further exploration. Furthermore, due to the low number of clinical samples, the expression of APBB2 in NSCLC, and the association between APBB2 expression and clinical features and prognosis of patients with NSCLC remains unknown. The underlying mechanisms of APBB2 in NSCLC will be further explored in future studies. Despite these limitations, the findings of the current study also provided some novel insights into our knowledge of NSCLC.

In conclusion, the present study demonstrated that miR-205-3p was significantly increased in NSCLC tissue and associated with the poor prognosis of patients with NSCLC. Mechanically, miR-205-3p promoted lung cancer progression by targeting APBB2. These results suggested that miR-205-3p may play a critical role in the development of NSCLC and it is of importance to further explore the biofunction of the miR-205-3p/APBB2 axis in NSCLC in future studies.

\section{Acknowledgements}

Not applicable.

\section{Funding}

This work was supported by The Shaanxi Provincial Key Research and Development Project (grant no. 2017SF-023).

\section{Availability of data and materials}

The datasets used and/or analyzed during the current study available from the corresponding author on reasonable request.

\section{Authors' contributions}

LBX and MQ conceived and designed the experiments. JX, YHZ, YD and XPR performed the experiments. JX, YJR, $\mathrm{DH}$ and SHW analyzed and interpreted the data. JX wrote the manuscript. LBX and MQ revised the manuscript. LBX,
JX and MQ confirm the authenticity of all the raw data. All authors have read and approved the final manuscript.

\section{Ethics approval and consent to participate}

Written informed consent was obtained from all patients and the study protocol was approved by the Ethics Committee of Shaanxi Provincial People's Hospital (Shaanxi, China). Animal care and study were approved by the Institutional Animal Care and Use Committee of Shaanxi Provincial People's Hospital.

\section{Patient consent for publication}

Not applicable.

\section{Competing interests}

The authors declare that they have no competing interests.

\section{References}

1. Bade BC and Dela Cruz CS: Lung cancer 2020: Epidemiology, etiology, and prevention. Clin Chest Med 41: 1-24, 2020.

2. Grimminger J, Richter M, Tello K, Sommer N, Gall H and Ghofrani HA: Thin Air resulting in high pressure: Mountain sickness and hypoxia-induced pulmonary hypertension. Can Respir J 2017: 8381653, 2017.

3. Ardila D, Kiraly AP, Bharadwaj S, Choi B, Reicher JJ, Peng L, Tse D, Etemadi M, Ye W, Corrado G, et al: End-to-end lung cancer screening with three-dimensional deep learning on low-dose chest computed tomography. Nat Med 25: 954-961, 2019.

4. Sutendra G and Michelakis ED: The metabolic basis of pulmonary arterial hypertension. Cell Metabolism 19: 558-573, 2014.

5. Parra V, Bravo-Sagua R, Norambuena-Soto I, Hernández-Fuentes CP, Gómez-Contreras AG, Verdejo HE, Mellado R, Chiong M, Lavandero S and Castro PF: Inhibition of mitochondrial fission prevents hypoxia-induced metabolic shift and cellular proliferation of pulmonary arterial smooth muscle cells. Biochim Biophys Acta Mol Basis Dis 1863: 2891-2903, 2017.

6. Farber HW and Loscalzo J: Pulmonary arterial hypertension. N Engl J Med 351: 1655-1665, 2004.

7. Aydin S, Kuloglu T, Aydin S, Kalayci M, Yilmaz M, Cakmak T, Albayrak S, Gungor S, Colakoglu N and Ozercan IH: A comprehensive immunohistochemical examination of the distribution of the fat-burning protein irisin in biological tissues. Peptides 61: 130-136, 2014

8. Lewis BP, Burge CB and Bartel DP: Conserved seed pairing, often flanked by adenosines, indicates that thousands of human genes are microRNA targets. Cell 120: 15-20, 2005.

9. Bueno MJ, Pérez de Castro I and Malumbres M: Control of cell proliferation pathways by microRNAs. Cell Cycle 7: 3143-3148, 2008.

10. Jovanovic $M$ and Hengartner MO: miRNAs and apoptosis: RNAs to die for. Oncogene 25: 6176-6187, 2006.

11. Wu F, Song H, Zhang Y, Zhang Y, Mu Q, Jiang M, Wang F, Zhang W, Li L, Li H, et al: Irisin induces angiogenesis in human umbilical vein endothelial cells in vitro and in zebrafish embryos in vivo via activation of the ERK signaling pathway. PLoS One 10: e0134662, 2015.

12. Chalmers S, Saunter C, Wilson C, Coats P, Girkin JM and McCarron JG: Mitochondrial motility and vascular smooth muscle proliferation. Arterioscler Thromb Vasc Biol 32: 3000-3011, 2012.

13. Li DJ, Li YH, Yuan HB, Qu LF and Wang P: The novel exercise-induced hormone irisin protects against neuronal injury via activation of the Akt and ERK1/2 signaling pathways and contributes to the neuroprotection of physical exercise in cerebral ischemia. Metabolism 68: 31-42, 2017.

14. Szarka RJ, Wang N, Gordon L, Nation PN and Smith RH: A murine model of pulmonary damage induced by lipopolysaccharide via intranasal instillation. J Immunol Methods 202: 49-57, 1997. 
15. Duan B, Guo T, Sun H, Cai R, Rui Q and Xi Z: miR-205 as a biological marker in non-small cell lung cancer. Biomed Pharmacother 91: 823-930, 2017.

16. Su N, Qiu H, Chen Y, Yang T, Yan Q and Wan X: miR-205 promotes tumor proliferation and invasion through targeting ESRRG in endometrial carcinoma. Oncol Rep 29: 2297-2302, 2013.

17. CaoH,PrattN,Mattison J,Zhao Y and Chang NS: Characterization of an apoptosis inhibitory domain at the C-temini of FE65-like protein. Biochem Biophys Res Commun 276: 843-850, 2000.

18. Goodman RB, Pugin J, Lee JS and Matthay MA Cytokine-mediated inflammation in acute lung injury. Cytokine Growth Factor Rev 14: 523-535, 2003.

19. Lim JH, Lee HJ, Ho Jung M and Song J: Coupling mitochondrial dysfunction to endoplasmic reticulum stress response: A molecular mechanism leading to hepatic insulin resistance. Cell Signal 21: 169-177, 2009.

20. Lim W, Ridge CA, Nicholson AG and Mirsadraee S: The 8th lung cancer TNM classification and clinical staging system: Review of the changes and clinical implications. Quant Imaging Med Surg 8: 709-718, 2018.

21. Livak KJ and Schmittgen TD: Analysis of relative gene expression data using real-time quantitative PCR and the 2(-Delta Delta C(T)) method. Methods 25: 402-408, 2001.

22. Kumar G, Goldberg SN, Wang Y, Velez E, Gourevitch S, Galun E and Ahmed M: Hepatic radiofrequency ablation: Markedly reduced systemic effects by modulating periablational inflammation via cyclooxygenase-2 inhibition. Eur Radiol 27: 1238-1247, 2017.

23. Reddy KB: MicroRNA (miRNA) in cancer. Cancer Cell Int 15 38. 2015.

24. Acunzo M, Romano G, Wernicke D and Croce CM: MicroRNA and cancer-a brief overview. Adv Biol Regul 57: 1-9, 2015.

25. EI-Awady RA,HersiF, AI-Tunaiji H,Saleh EM, Abdel-Wahab AH, Al Homssi A, Suhail M, El-Serafi A and Al-Tel T: Epigenetics and miRNAs as predictive markers and targets for lung cancer chemotherapy. Cancer Biol Ther 16: 1056-1070, 2015.

26. Markou A, Zacridou M and Lianidou ES: $m i R-21$ as a novel therapeutic target in lung cnacer. Lung Cancer (Auckl) 7: 19-27, 2016.
27. Nana-Sinkam SP and Croce CM: Clinical applications for microRNAs in cancer. Clin Pharmacol Ther 93: 98-104, 2013.

28. Wang M, Wang J, Liu Y, Wang J, Nie Y, Si B, Liu Y, Wang X, Chen S, Hei TK, et al: Subcellular targets of zinc oxide nanoparticles during the aging process: Role of cross-talk between mitochondrial dysfunction and endoplasmic reticulum stress in the genotoxic response. Toxicol Sci: Jun 7, 2019 doi: 10.1093/toxsci/kfz132 (Epub ahead of print).

29. Mazur-Bialy AI: Irisin acts as a regulator of macrophages host defense. Life Sci 176: 21-25, 2017.

30. Park DW, Jiang S, Liu Y, Siegal GP, Inoki K, Abraham E and Zmijewski JW: GSK3 $\beta$-dependent inhibition of AMPK potentiates activation of neutrophils and macrophages and enhances severity of acute lung injury. Am J Physiol Lung Cell Mol Physiol 307: L735-L745, 2014.

31. Batty D, Rapic'-Otrin V,Levine AS and Wood RD: Stable binding of human XPC complex to irradiated DNA confers strong discrimination for damaged sites. J Mol Biol 300: 275-290, 2000.

32. Hanna JA, Hahn L, Agarwal S and Rimm DL: In situ measurement of miR-205 in malignant melanoma tissue supports its role as a tumor suppressor microRNA. Lab Invest 92: 1390-1397, 2012.

33. Rubenfeld GD, Caldwell E, Peabody E, Weaver J, Martin DP, Neff M, Stern EJ and Hudson LD: Incidence and outcomes of acute lung injury. N Engl J Med 353: 1685-1693, 2005.

34. Niu K, Shen W, Zhang Y, Zhao Y and Lu Y: MiR-205 promotes motility of ovarian cancer cells via targeting ZEB1. Gene 574: 330-336, 2015.

35. Golanska E, Sieruta M, Gresner SM, Pfeffer A, ChodakowskaZebrowska M, Sobow TM, Klich I, Mossakowska M, Szybinska A, Barcikowska M and Liberski PP: APBB2 genetic polymorphisms are associated with severe cognitive impairment in centenarians. Exp Gerontol 48: 391-394, 2013.

(†) $\odot$ This work is licensed under a Creative Commons Attribution-NonCommercial-NoDerivatives 4.0 International (CC BY-NC-ND 4.0) License. 